Dermatologische Zeitschrift. 1922;35:I-IV

\title{
Contents, Vol. 35, 1922
}

Inhaltsverzeichnis.

Originalarbeiten. Seite

Baer, L·, Über die einzeitig kombinierte intravenose Neo-

salvarsan-Novasurol-Behandlung (nach Bruck-Becher) 43 Bergel, S., Die biologisch-klinische

Bedeutung der Lympho-

zyten für die Syphilis 279

Boas, H, Unfall als Ursache von Acrodermatitis atrophicans 99 Brünauer, Stef. Rob.,

Epidermophytia inguinahs genera-

lisata mit Beteiligung der Nägel 148

Gutmann, C, Über sogenannte ,,fixe" und allgemeine urti-

karielle, rezidivierende Exantheaie nach Salvarsan und

Quecksilber 135

Haxthausen, H., Untersuchungen über die Pathogenese der

Jod- und Bromäkne 54

Heller, J u 1. , Zur Kasuistik seltener Nagelerkrankungen.

Nachtrag zu XVI: Chemisehe Untersuchung des Falles . 288 Hoffmann, Erich, Über häufigeres

Vorkommen von Ikterus

bei Syphilis und von Ikterus überhaupt 362

Hoíraann, E. und O· Mergelsberg, Zehn Jahre Salvarsan-

therapie an der Bonner Hautklinik 1

Klöppel, F. W. , Zur Kenntnis des gonorrhöischen Fersen-

schmerzes 90

Kyrle, J., Die Bedeutung des unspezifischen Heilfaktors in

der Syphilistherapie 313

Langer, Erich, Karzinom und Trauma 212

Lippert, H., Über den Morbus Darier. Mit Mitteilung eines

Falles 76

Mayr, Julius K., Die umschriebene Yerminderung des Gleich-

stromwiderstandes der Haut. Erste Mitteilung .... 332 Müller, H., Neurodermitis und

Klitoriskarzinom bei jungem

Mädchen 70

Nathan, E., und H.Martin, Quantitative Bestimmung der

Reagine des Syphilisserums mittels Ausflockung und ihre

Bedeutung für die Serodiagnose und Salvarsantherapie

der Syphilis 189

IV Inlialts $\lambda$ erzeichnis.

Seite

S c h o 11 z, W., Über Protoplasinaaktivierung und Osmotherapie, insbesondere durch intravenöse Traubenzuckerinjektionen 127 
Zurhelle, Emil, Über den Anteil feinster Bindegewebsfibrillen, der sogen. Gitterfasern, am Aufbau syphilitischer und anderer Hauteffloreszenzen, gleichzeitig ein Beitrag zu ihrer Konsistenz, insbesondere zur Härte des Primäraffektes 251

-, Ein Fall von ausgedehntem Leukoderm bei Parapsoriasis guttata 339

Felix Lewandowsky $\uparrow 154$

Gesellschaftsberichte.

Berliner Dermatologische Gesellschaft. Sitzung vom 12. IV., 10. V. und 14. VI. $1921 \quad 163,220,293$

Vereinigung Rheinisch-westfälischer Dermatologen. Sitzung in Elberfeld am 5. November $1921 \quad 342$

Versammlung südwestdeutscher Dermatologen vom 8.-9. Oktober 1921 zu Frankfurt a. M . . 150

Dänische Dermatologische Gesellschaft. Sitzungen vom 5. XL, 4. XII. 1919 und 4. II. 3. III. und 7. IV. 1920 . . 99, 290 Moskauer venerologisclidermatologische Gesellschaft. Sitzungen

vom 3. April, 15. Mai und 5. Juni 1921

Periodische Literatur 106, 175, 234, 306, 345

Buchbesprechungen 123, 188, 248, 311

Perso $\pi$ alien und Tagesnachrichten .... 126, 250, 312, 363

Sach- und Namenregister $\quad 364,368$

Berichtigung zu Band XXXIV, Heft 3/4, S. $208 \quad 126$ 